\title{
Multi-CFD Timing Estimators for PET Block Detectors
}

\author{
M. G. Ullisch and W. W. Moses, Fellow, IEEE
}

\begin{abstract}
In a conventional PET system with block detectors, a timing estimator is created by generating the analog sum of the signals from the four photomultiplier tubes (PMT) in a module and discriminating the sum with a single constant fraction discriminator (CFD). The differences in the propagation time between the PMTs in the module can potentially degrade the timing resolution of the module. While this degradation is probably too small to affect performance in conventional PET imaging, it may impact the timing inaccuracy for time-of-flight PET systems (which have higher timing resolution requirements). Using a separate CFD for each PMT would allow for propagation time differences to be removed through calibration and correction in software.

In this paper we investigate and quantify the timing resolution achievable when the signal from each of the 4 PMTs is digitized by a separate CFD. Several methods are explored for both obtaining values for the propagation time differences between the PMTs and combining the four arrival times to form a single timing estimator. We find that the propagation time correction factors are best derived through an exhaustive search, and that the "weighted average" method provides the best timing estimator. Using these methods, the timing resolution achieved with 4 CFDs $(1052 \pm 82 \mathrm{ps})$ is equivalent to the timing resolution with the conventional single CFD setup (1067 $\pm 158 \mathrm{ps})$.
\end{abstract}

Keywords-timing resolution, time of flight, PET, constant fraction discriminator, CFD.

\section{INTRODUCTION}

Virtually all commercial PET cameras use block detectors $[1,2]$ in which a relatively large number of individual scintillator crystals (typically 50-150) are read out with a small number of PMTs (typically 4). A timing estimator is generated via an analog sum of the four PMT signals, then converting this analog signal into a digital timing signal using a CFD [3-8]. Differences in the propagation times between the 4 PMTs affect the leading edge of the summed signal and thus affect the timing resolution. Previous work [9] has shown this contribution to be 250 ps to 500 ps fwhm. In conventional PET systems with timing resolutions on the order of nanoseconds this is insignificant, but in time-offlight (TOF) PET systems with sub-nanosecond timing

Manuscript received December 10,2005. This work was supported in part by the U.S. Department of Energy under contract No. DE-AC0205CH11231, and in part by Public Health Service Grant No. R33-EB001928.

W. W. Moses is with Lawrence Berkeley National Laboratory, Berkeley, CA 94720 USA (telephone: ++1-510-486-4432, e-mail: wwmoses@lbl.gov).

M. Ullisch is with Lawrence Berkeley National Laboratory, Berkeley, CA 94720 USA (e-mail: marcus@ullisch.de). resolutions [10-23] this can be an appreciable source of timing jitter.

These part-to-part variations in the propagation time could be corrected in the manufacturing process either through preselecting PMTs based on their propagation delay or with custom-length cables for each PMT. However, these approaches are inflexible and are difficult or impossible to recalibrate. A more flexible approach is to discriminate each PMT with its own CFD and correct for the propagation time differences of the PMTs in software. This also allows different software algorithms for generating the single timing estimator out of the four individual timing signals. This approach is cost effective, as the costs for the three additional CFDs per module are small compared to other production costs.

The purpose of this paper is to investigate whether an improvement of the timing resolution is achievable when four CFDs are used instead of one.

\section{MATERIALS AND MethodS}

To measure the timing resolution of a detector module we have constructed a test system replacing the relevant timing electronics (CFD and TDC) of a PET system with high performance NIM components.

\section{A. Trigger Signal / Reference Detector}

A reference detector is constructed, consisting of a $10 \mathrm{~mm}$ cube of $\mathrm{BaF}_{2}$ scintillator coupled to a Hamamatsu H-5321 PMT assembly operated at $-2300 \mathrm{~V}$ and read out with one channel of a Canberra 454 NIM CFD. A pair of identical reference detectors is excited with coincident $511 \mathrm{keV}$ annihilation photons from a $120 \mu \mathrm{Ci}{ }^{68} \mathrm{Ge}$ source, and the time difference of each event measured with an Ortec 556 NIM TAC and a National Instruments PXI-7831R 16-bit ADC read out by a personal computer. This measurement yields 212 ps fwhm coincidence timing resolution, implying a trigger accuracy of $150 \mathrm{ps}$ fwhm. The electronics accuracy is estimated by triggering both CFDs with the same input signal, yielding a timing resolution of 25 ps fwhm $(<2$ ADC bins). One $\mathrm{BaF}_{2}$ module and the $120 \mu \mathrm{Ci}{ }^{68} \mathrm{Ge}$ source are used to generate reference triggers for all of the subsequent measurements presented in this work.

\section{B. Detector Module}

A detector module from the initial version of the CPS Accel [24] whole body PET camera is used. It is approximately $50 \mathrm{~mm}$ square and consists of an $8 \times 8$ array of 


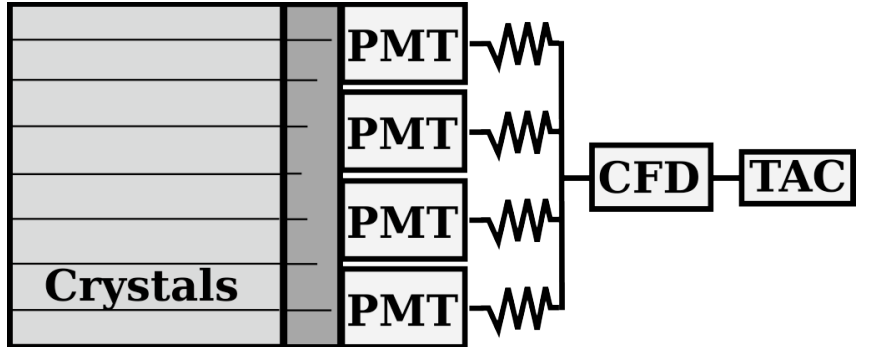

Figure 1: In the conventional single-CFD setup the outputs of the 4 PMTs in a module are summed (via $22 \Omega$ resistors) and sent to a single CFD.

$6.75 \times 6.75 \times 25 \mathrm{~mm}^{3}$ LSO [25-27] scintillator crystals read out with 4 Hamamatsu R8619-02 PMTs.

To mimic conventional PET readout, the four PMTs of the module are connected to a single channel of a Canberra 454 NIM CFD through $22 \Omega$ resistors (fig. 1). Adding this resistor does not change the timing resolution. While the RMS propagation time variation of these four PMTs is $108 \mathrm{ps}$, no attempt is made to correct for the different PMT propagation times. At this stage, the bases of each PMT are adjusted such that each PMT has the same gain. An LSO crystal is coupled to one of the PMTs, a $511 \mathrm{keV}$ excited pulse height spectrum is obtained, the base adjusted so the photopeak lies in a specific channel, and the process repeated for all four PMTs.

For the 4 CFD experiments, the same detector module is used but the electronics is changed so that each PMT is connected directly to a separate CFD channel and digitized with a separate TAC (fig. 2). Each PMT output is also sent to a Cremat CR-501 shaper amplifier with 500 ns shaping time and the four pulse heights are digitized with four ADCs. For each trigger, all four times (i.e., TAC outputs) and all four pulse heights (i.e., shaper amplifier outputs) are digitized and read out by a personal computer.

Two hours of data are acquired for each crystal and split up into two independent datasets. All odd numbered events are used with the optimization routine described later and all even numbered events are used to generate the timing measurements presented in table 1 . The acquired data is then processed with different algorithms implemented in LabView which are described in the following sections.

\section{MEASUREMENTS}

\section{A. Single-CFD}

Electronic collimation is used to acquire coincidence events from an area of the block that is approximately $10 \mathrm{~mm}$ x $10 \mathrm{~mm}$ and centered on an individual crystal in the array. The timing resolution for each of the 64 crystals is measured. To reduce the statistical errors, data is acquired for one hour for each crystal. The timing information from these events is histogrammed and the timing resolution for each crystal computed. The average timing resolution for the 64 crystals is $1067 \mathrm{ps}$ fwhm with an uncertainty of $158 \mathrm{ps}$, where the uncertainty is the RMS variation in the timing resolution for the 64 crystals. These data are shown in Table 1 .

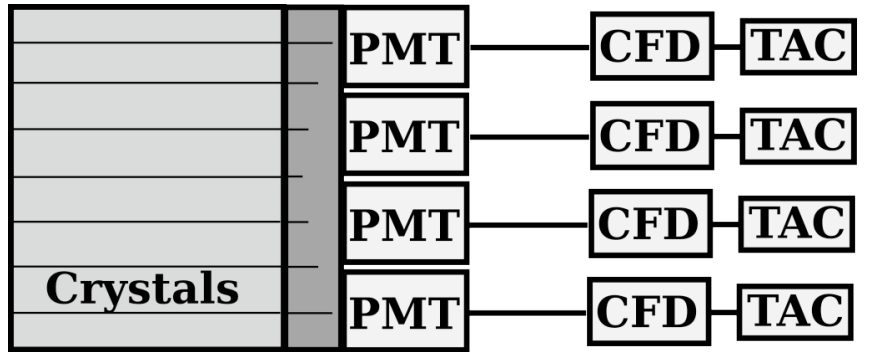

Figure 2: In the investigated 4 CFD setup the output of each PMT in a module is digitized by a separate CFD. Propagation time correction and timing estimator generation are undertaken in software.

\section{B. Multi-CFD}

When using the multi-CFD system, two things must be done in order to obtain a timing estimator for each event. First, the difference in propagation times must be corrected for in software by adding a correction factor to the timing value from each PMT. Then the four corrected PMT timing values are used to generate a single timing estimator for the module. There are, however, several ways to obtain the propagation time differences, and several ways to combine the four timing values to create a timing estimator. In this section, we describe the different methods we have used and the performance of each method.

1) Propagation Time Correction

We first obtain the additive correction factor for the four timestamps acquired in each event. Four different methods to derive these correction factors are investigated:

a) Single Crystal. A single $6 \times 6 \times 25 \mathrm{~mm}^{3}$ LSO crystal is coupled to one of the PMTs. A reference detector is used to measure coincidence events, the arrival times are histogrammed, and the centroid of the timing distribution measured. The process is repeated for each of the four PMTs. Correction factors are chosen such that the centroids of the distributions are aligned. The same correction factors are used for all 64 crystals.

b) Single Photoelectron Transit Time. A diode laser with a narrow ( $60 \mathrm{ps}$ fwhm) pulse is used to excite one of the PMTs [19]. The laser beam is optically attenuated so that the average number of generated photoelectrons per pulse is much less than one. The arrival times (with respect to the excitation pulse) are histogrammed, and the centroid of the timing distribution (i.e., the single photoelectron transit time of the PMT) measured. The process is repeated for each of the four PMTs. Correction factors are chosen such that the centroids of the distributions are aligned. The same correction factors are used for all 64 crystals.

c) Block Detector. The Accel scintillator block described earlier is coupled to the four PMTs in the module. To ensure equal length light path lengths from the excitation position to each PMT, the module is excited at the center of the scintillator block. Arrival times are histogrammed, centroids are determined, and correction factors obtained using the same procedure as used for the Single Crystal method. The same correction factors are used for all 64 crystals.

d) Iterative Optimization. A scintillator block is coupled to the four PMTs in the module and timing data is collected. 
Table 1: Timing Resolution of the Accel Detector Module with the conventional single-CFD timing estimator and multi-CFD timing estimator using different propagation time correction techniques for the part-to-part variation of the propagation time in the PMTs. The Iterative Optimization Procedure is used to calculate both crystal-independent (CI) and crystaldependent (CD) correction factors.

\begin{tabular}{|l|c|c|c|}
\hline Algorithm & $\begin{array}{c}\text { Propagation Time } \\
\text { Correction }\end{array}$ & $\begin{array}{c}\text { Timing } \\
\text { Resolution } \\
\text { FWHM } \\
\text { (ps) }\end{array}$ & $\begin{array}{c}\text { Relative } \\
\text { Timing } \\
\text { Resolution }\end{array}$ \\
\hline Single CFD & Not Applicable & $1067 \pm 158$ & $1.00 \pm 0.15$ \\
\hline Weig. Avg. & $\begin{array}{c}\text { Iterative Optim. } \\
\text { (CD) }\end{array}$ & $1052 \pm 82$ & $0.99 \pm 0.08$ \\
\hline Weig. Avg. & $\begin{array}{c}\text { Iterative Optim. } \\
\text { (CI) }\end{array}$ & $1117 \pm 88$ & $1.05 \pm 0.08$ \\
\hline Weig. Avg. & Single Crystal & $1126 \pm 84$ & $1.06 \pm 0.08$ \\
\hline Weig. Avg. & Block & $1232 \pm 181$ & $1.15 \pm 0.17$ \\
\hline Weig. Avg. & No Correction & $1128 \pm 109$ & $1.06 \pm 0.10$ \\
\hline First Signal & $\begin{array}{c}\text { Iterative Optim. } \\
\text { (CD) }\end{array}$ & $1152 \pm 107$ & $1.08 \pm 0.10$ \\
\hline First Signal & $\begin{array}{c}\text { Iterative Optim. } \\
\text { (CI) }\end{array}$ & $1184 \pm 104$ & $1.11 \pm 0.10$ \\
\hline First Signal & Single Crystal & $1177 \pm 105$ & $1.10 \pm 0.10$ \\
\hline First Signal & Block & $1419 \pm 414$ & $1.33 \pm 0.39$ \\
\hline First Signal & No Correction & $1330 \pm 253$ & $1.25 \pm 0.24$ \\
\hline Max. Ampl. & $\begin{array}{c}\text { Iterative Optim. } \\
\text { (CD) }\end{array}$ & $1240 \pm 135$ & $1.16 \pm 0.13$ \\
\hline Max. Ampl. & $\begin{array}{c}\text { Iterative Optim. } \\
\text { (CI) }\end{array}$ & $1237 \pm 144$ & $1.16 \pm 0.13$ \\
\hline Max. Ampl & Single Crystal & $1283 \pm 148$ & $1.20 \pm 0.14$ \\
\hline Max. Ampl. & Block & $1329 \pm 257$ & $1.25 \pm 0.24$ \\
\hline Max. Ampl. & No Correction & $1445 \pm 437$ & $1.35 \pm 0.41$ \\
\hline
\end{tabular}

Using an exhaustive search, different delays are tried and the resulting timing resolution is minimized. To prevent bias, different sets of events are used for obtaining the calibration factors (i.e., the relative delays) and comparing the algorithms. While the previous methods for obtaining the delay factors do not depend on how the timing estimator is generated, this method depends on the estimator used. Unlike the first three methods, this method is able to utilize information on which crystal the interaction is assigned to. We use this method to derive both crystal-independent and crystal dependent PMT correction factors.

\section{2) Timing Estimator Algorithm}

Several different algorithms are explored for combining, for each event, the four measured times into a single timing estimator. All the algorithms use the same set of events to measure the resulting coincidence timing resolution, all use data that are corrected for propagation delay variations, and all have the pulse height data from each PMT available. The algorithms that were investigated are:

a) First Signal. The timing data from the first PMT to fire is used as the estimator.

b) Second, Third, \& Fourth Signal. For the Second Signal algorithm, the Third Signal algorithm, and the Fourth Signal algorithm, the timing data from the second, third, or fourth PMT to fire is used as the estimator.

c) Maximum Amplitude. The timing data from the PMT with the highest signal amplitude is used as the estimator. d) Weighted Average. The timing data from each PMT is weighted by the pulse height for that PMT and the linear weighted average used as the estimator.

3) Results

The delay correction factors obtained by the Single Photoelectron Transit Time measurements were not significantly different than those obtained by the Single Crystal method. Therefore, for simplicity we have omitted these results from Table 1. In addition, the Second, Third, and Fourth Signal algorithms gave significantly worse results than any of the other algorithms, and so their results are also not shown. The remaining results of the different combinations of propagation time correction factors (None, Single Crystal, Block, Crystal-Dependent and CrystalIndependent Iterative Optimization) and timing generator algorithm (Weighted Average, First Signal and Maximum Amplitude) are shown in Table 1.

\section{DISCUSSION}

Of the three algorithms explored using the 4-CFD setup, the Weighted Average algorithm $(1052 \pm 82)$ outperformed the First Signal algorithm $(1152 \pm 107 \mathrm{ps})$ by $10 \%$ and the Maximum Amplitude algorithm (1240 135 ps) by $18 \%$.

It is not a priori clear which algorithm should give the best performance. It can be demonstrated [28-30] that if the individual photoelectrons in the leading edge of the signal are resolved in time, the First Signal algorithm should have the best performance. However, the photoelectron rate from $511 \mathrm{keV}$ interactions in LSO is high enough that individual photoelectrons are not resolved. The Weighted Average algorithm has the advantage of creating the estimator by using all available information (i.e. all four PMT signals) while the First Signal and Maximum Amplitude algorithms only use a fourth of the information (i.e., the signal from a single PMT).

In the 4-CFD setup, propagation time correction significantly improves the timing resolution (as compared to not using additive propagation time correction factors). The Iterative Optimization method produces the best timing resolution with an improvement of $13-14 \%$ for both the First Signal and the Maximum Amplitude estimator algorithms and $7 \%$ for the Weighted Average algorithm.

The Single Crystal method achieves an improvement of $11 \%$ for the First Signal and Maximum Amplitude algorithms, which is only slightly worse than the Iterative Optimization. For the Weighted Average algorithm it does not show a significant change of the timing resolution. The Block method shows the worst performance, improving the timing resolution by $8 \%$ with the Maximum Amplitude estimator but degrading the timing resolution by $6 \%$ and $9 \%$ with the First Signal Weighted Average estimator.

The relatively poor performance of the Block method is partially explained by the relatively poor timing resolution with a block detector. As shown in [9] a scintillator array has a significantly worse timing resolution than a single crystal, caused by a lower light-output of the crystal array. Thus the 
correction factors derived using measurements from the block have larger errors. The other methods that directly measure the propagation delay (Single Crystal and Single Photoelectron Transit Time) appear to measure the delay more precisely, and so have nearly identical measured delay values and performance.

Unlike the other methods, the Iterative Optimization method does not attempt to measure the propagation delay but uses whichever values for the relative delays provide the best performance. One implication is that since the delay values are not tied to a PMT-based measurement, a crystaldependent value for each PMT's propagation delay can be used. This is justified, as the transit time in a PMT usually depend on the position on the photocathode where the photoelectron is generated, and light path variations between different crystals in the block are likely.

The similar results of the Single Crystal routine and the Crystal-Independent optimization for all three estimator algorithms suggest that the two methods are equivalent. For the Maximum Amplitude and First Signal algorithm the Crystal-Dependent optimization does not show any significant advantage ( $0 \%-2 \%)$ over the Crystal-Independent optimization. However, it is the only correction method that significantly improves $(7 \%)$ the timing resolution of the Weighted Average algorithm.

The best timing resolution we achieved with the 4 CFD setup $(1052 \pm 82$ ps with Weighted Average, CrystalDependent optimization) is nearly identical to the timing resolution measured with the conventional single CFD setup $(1067 \pm 158 \mathrm{ps})$. Again, it is not a priori clear whether one setup should outperform the other. In the limit of individually resolved photoelectrons, the 4-CFD system with First Signal algorithm should have identical performance as the single CFD system (if propagation delays are not corrected for), but photoelectrons are not individually resolved in our apparatus.

Finally, we note that care should be taken when extending the conclusion drawn from this system to other systems. The timing resolution depends on many factors, and the measurements made herein do not represent all systems. In particular, the transit time variations between photomultiplier tubes are a small fraction of the overall timing resolution (108 ps RMS transit time variation compared to $\sim 1.1 \mathrm{~ns}$ fwhm coincidence timing resolution), so our measurements are relatively insensitive to the methods used to correct for transit time variation. For TOF PET systems that have better timing resolution [21-23,31], these corrections will be much more important. However, we believe that they reasonably represent existing LSO-based PET detector modules.

\section{CONCLUSIONS}

We have instrumented a commercial LSO-based PET detector module with a CFD/TDC combination on each of its four photomultiplier tubes. The best timing performance for this 4-CFD setup is a resolution of $1052 \pm 82 \mathrm{ps,} \mathrm{which} \mathrm{is}$ equal to the conventional single CFD method ( $1067 \pm 158 \mathrm{ps})$. We explored several algorithms for combining the four timing measurements to obtain the optimal timing estimator.
We found that the Weighted Average estimator outperforms the First Signal estimator (1152 $\pm 107 \mathrm{ps})$ and the Maximum Amplitude estimator (1254 $\pm 134 \mathrm{ps})$.

Several different methods were explored for determining the propagation time differences between the four PMTs. We found that an Iterative Optimization method has the best performance, and slightly out-performs the Single Crystal method. Both out-perform the Block method. Using propagation time correction improves the timing resolution by up to $14 \%$, depending on the algorithm used.

\section{ACKNOWLEDGMENTS}

We thank Dr. Michael Casey and Dr. Bernard Bendriem of CPS Innovations for supplying invaluable components and information. This work was supported in part by the Director, Office of Science, Office of Biological and Environmental Research, Medical Science Division of the U.S. Department of Energy under Contract No. DE-AC02-05CH11231, and in part by the National Institutes of Health, National Institute of Biomedical Imaging and Bioengineering under grant No. R33-EB001928. Reference to a company or product name does not imply approval or recommendation by the University of California or the U.S. Department of Energy to the exclusion of others that may be suitable.

\section{REFERENCES}

[1]M. E. Casey and R. Nutt, "A multicrystal two dimensional BGO detector system for positron emission tomography," IEEE Trans. Nucl. Sci., vol. NS-33, pp. 460-463, Feb. 1986.

[2] W. H. Wong, J. Uribe, K. Hicks and M. Zambelli, "A 2-dimensional detector decoding study on BGO arrays with quadrant sharing photomultipliers," IEEE Trans. Nucl. Sci., vol. 41, pp. 1453-1457, Aug. 1994.

[3]D. A. Gedcke and W. J. McDonald, "A constant fraction of pulse height trigger for optimum time resolution," Nucl. Instr. Meth., vol. 55, pp. $377-380,1967$.

[4]D. A. Gedcke and W. J. McDonald, "Design of the constant fraction of pulse height trigger for optimum time resolution," Nucl. Instr. Meth., vol. 58, pp. 253-260, 1968.

[5]D. M. Binkley, M. L. Simpson, and J. M. Rochelle, "A monolithic, $2 \mu \mathrm{m}$ CMOS constant-fraction discriminator for moderate time resolution systems," IEEE Trans. Nucl. Sci., vol. 38, pp. 1754-1759, Dec. 1991.

[6]D. M. Binkley, "Performance of non-delay-line constant-fraction discriminator timing circuits," IEEE Trans. Nucl. Sci., vol. 41, pp. 1169-1175, Aug. 1994.

[7]D. M. Binkley, B. S. Puckett, B. K. Swann, J. A. Rochelle, M. S. Musrock, et al., "A 10-mc/s, 0.5- $\mu \mathrm{m}$ CMOS constant-fraction discriminator having built-in pulse tail cancellation," IEEE Trans. Nucl. Sci., vol. NS-49, pp. 1130-1140, June 2002.

[8]B. K. Swann, J. M. Rochelle, D. M. Binkley, B. S. Puckett, B. J. Blalock, et al., "A custom mixed-signal CMOS integrated circuit for high performance PET tomograph front-end applications," IEEE Trans. Nucl. Sci., vol. NS-50, pp. 909-914, Aug. 2003.

[9] W. W. Moses and M. Ullisch, "Factors influencing timing resolution in a commercial LSO PET camera," IEEE Trans. Nucl. Sci., vol. NS-52, (accepted for publication), 2005.

[10] M. M. Ter-Pogossian, D. C. Ficke, M. Yamamoto and J. T. Hood. Design characteristics and preliminary testing of Super-PETT I, a positron emission tomograph utilizing photon time-of-flight information (TOF PET). Proceedings of The Workshop on Time of Flight Tomography, pp. 37-41, (St. Louis, MO, May 1982.

[11] M. M. Ter-Pogossian, D. C. Ficke, M. Yamamoto and J. T. Hood, "Super PETTI: A positron emission tomograph utilizing photon timeof-flight information," IEEE Trans. Med. Img., vol. MI-1, pp. 179-187, Nov. 1982. 
[12] N. A. Mullani, W. H. Wong, R. K. Hartz, K. Yerian, E. A. Philippe, et al. Design of TOFPET: a high resolution time-of-flight positron camera. Proceedings of The Workshop on Time of Flight Tomography, pp. 31-36, (St. Louis, MO, May 1982.

[13] R. Gariod, R. Allemand, E. Cormoreche, M. Laval and M. Moszynski. The LETI positron tomograph architecture and time of flight improvements. Proceedings of The Workshop on Time of Flight Tomography, pp. 25-29, (Edited by St. Louis, MO, May 1982.

[14] T. F. Budinger, "Time-of-flight positron emission tomography: Status relative to conventional PET," J. Nucl. Med., vol. 24, pp. 73-78, 1983.

[15] W. H. Wong, N. A. Mullani, E. A. Phillipe, R. Hartz and K. L. Gould, "Image improvement and design optimization of the time-of-flight PET," J. Nucl. Med., vol. 24, pp. 52-60, Jan. 1983.

[16] T. K. Lewellen, A. N. Bice, R. L. Harrison, M. D. Pencke and J. M. Link, "Performance measurements of the SP3000/UW time-of-flight positron emission tomograph," IEEE Trans. Nucl. Sci., vol. NS-35, pp. 665-669, Feb. 1988.

[17] B. Mazoyer, R. Trebossen, C. Schoukroun, B. Verrey, A. Syrota, et al., "Physical characteristics of TTV03, a new high spatial resolution timeof-flight positron tomograph," IEEE Trans. Nucl. Sci., vol. NS-37, pp. 778-782, Apr. 1990.

[18] M. M. Ter-Pogossian, D. C. Ficke, D. E. Beecher, G. R. Hoffmann and S. R. Bergmann, "The Super PET 3000-E - a PET scanner designed for high count rate cardiac applications," J. Comput. Assist. Tomogr., vol. 18, pp. 661-669, July 1994.

[19] W. W. Moses and S. E. Derenzo, "Prospects for time-of-flight PET using LSO scintillator," IEEE Trans. Nucl Sci., vol. NS-46, pp. 474478, June 1999.

[20] W. W. Moses, "Time of flight in PET revisited," IEEE Trans. Nucl. Sci., vol. NS-50, pp. 1325-1330, Oct. 2003.

[21] A. Kuhn, S. Surti, J. S. Karp, G. Muehllehner, F. M. Newcomer, et al. Performance assessment of pixellated $\mathrm{LaBr}_{3}$ detector modules for TOF PET. Proceedings of The IEEE 2004 Nuclear Science Symposium, pp. M9-59, (Edited by A. Siebert), Rome, Italy, 2004.

[22] S. Surti, J. S. Karp, and G. Muehllehner. Image quality assessment of $\mathrm{LaBr}_{3}$ based 3D PET scanners. Proceedings of The IEEE 2003 Nuclear Science Symposium and Medical Imaging Conference, pp. M3-148, (Edited by S. D. Metzler), Portland, OR, 2003.

[23] S. Surti, J. S. Karp, L. M. Popescu, M. E. Daube-Witherspoon and M. Werner. Investigation of image quality and NEC in a TOF-capable PET scanner. Proceedings of The IEEE 2004 Nuclear Science Symposium, pp. M10-276, (Edited by A. Siebert), Rome, Italy, 2004.

[24] T. J. Spinks and P. M. Bloomfield. A comparison of count rate performance for ${ }^{15} \mathrm{O}$-water blood flow studies in the CTI HR+ and Accel tomographs in 3D mode. Proceedings of The IEEE 2002 Nuclear Science Symposium and Medical Imaging Conference, $\mathrm{pp}$. M10-59, (Edited by S. Metzler), Norfolk, VA, 2002.

[25] C. L. Melcher and J. S. Schweitzer, "Cerium-doped lutetium orthosilicate: a fast, efficient new scintillator," IEEE Trans. Nucl. Sci., vol. NS-39, pp. 502-505, Aug. 1992.

[26] C. L. Melcher, M. Schmand, M. Eriksson, L. Eriksson, M. Casey, et al., "Scintillation properties of LSO:Ce boules," Nuclear Science, IEEE Transactions on, vol. 47, pp. 965-968, June 2000.

[27] C. L. Melcher, M. A. Spurrier, M. J. Schmand, L. A. Eriksson and R. Nutt, "Advances in the scintillation performance of LSO:Ce single crystals," IEEE Trans. Nucl. Sci., vol. NS-50, pp. 762-766, Aug. 2003.

[28] R. F. Post and L. I. Schiff, "Statistical limitations on the resolving time of a scintillation counter," Phys. Rev., vol. 80, pp. 1113-1120, Dec. 1950.

[29] L. G. Hyman, R. M. Schwarcz, and R. A. Schluter, "Study of high speed photomultiplier tube systems," Rev. Sci. Instr., vol. 35, pp. $393-$ 406, Mar. 1964.

[30] L. G. Hyman, "Time resolution of photomultiplier tube systems," Rev. Sci. Instr., vol. 36, pp. 193-196, Feb. 1965.

[31] A. Kuhn, S. Surti, J. S. Karp, P. S. Raby, K. S. Shah, et al., "Design of a lanthanum bromide detector for time-of-flight PET," IEEE Trans. Nucl. Sci., vol. NS-51, pp. 2550-2557, Oct. 2004. 